\title{
A Preliminary Report of Predisposing Factors and Predominant Microbiological Diagnosis of Corneal Ulcers Seen at the Federal Teaching Hospital Abakaliki, Nigeria
}

\author{
Chimdia E. Ogbonnaya ${ }^{1}$, Favoured Walter-Ugwuocha ${ }^{2}$, Edak Ezeanosike ${ }^{1}$, Chinyelu N. Ezisi' ${ }^{1}$, \\ Boniface N. Ukwah ${ }^{3}$, Helen A. Ginger-Eke ${ }^{1}$ \\ ${ }^{1}$ Department of Ophthalmology, Ebonyi State University, Abakaliki, Nigeria \\ ${ }^{2}$ Department of Medical Laboratories, Zankli Hospital, Abuja, Nigeria \\ ${ }^{3}$ Department of Medical Laboratory Sciences (Medical Microbiology Unit), Faculty of Health Science and Technology, Ebonyi \\ State University, Abakaliki, Nigeria \\ Email: *chimdiaogbonnaya@yahoo.com
}

How to cite this paper: Ogbonnaya, C.E., Walter-Ugwuocha, F.., Ezeanosike, E., Ezisi, C.N., Ukwah, B.N. and Ginger-Eke, H.A. (2019) A Preliminary Report of Predisposing Factors and Predominant Microbiological Diagnosis of Corneal Ulcers Seen at the Federal Teaching Hospital Abakaliki, Nigeria. Open Journal of Ophthalmology, 9, 94-104.

https://doi.org/10.4236/ojoph.2019.92011

Received: February 2, 2019

Accepted: May 21, 2019

Published: May 24, 2019

Copyright $\odot 2019$ by author(s) and Scientific Research Publishing Inc. This work is licensed under the Creative Commons Attribution International License (CC BY 4.0).

http://creativecommons.org/licenses/by/4.0/ (c) (i) Open Access

\begin{abstract}
Background: Microbial keratitis often results in poor visual outcome despite treatment. A revision of treatment protocol based on local evidence may be required in order to obtain better treatment outcome. Objectives: To determine the predisposing factors and predominant microbiological diagnosis of corneal ulcers seen at the Federal Teaching Hospital Abakaliki (FETHA), Ebonyi State, Nigeria. Materials and Methods: This is a preliminary report of an on-going longitudinal descriptive study of all consenting corneal ulcer patients managed at the FETHA eye clinic over a 4-month period. Information obtained were socio-demographic data, presenting complaints, duration of symptoms prior to presentation, history of preceding trauma, medications used before presentation, presenting and final visual acuity and microbiological diagnosis. Results: A diagnosis of corneal ulcer was made in 8 out of the 852 outpatients seen over the study period giving a hospital prevalence rate of $0.59 \%$. Five patients $(62.50 \%)$ were males, five $(62.50 \%)$ were farmers and 4 patients (50\%) were above 60 years of age. The microbial diagnoses were bacterial keratitis 37.5\% (Staphylococcus aureus), fungal keratitis 25\% (Fusarium spp. and aspergillus) and acanthamoeba (25\%). None of the patients ever used contact lenses. There was a history of eye trauma in $50 \%$ of the patients. All the eyes presented blind after a period of failed attempts to treat by self or quacks. Mean duration before presentation was two weeks. Treatment improved the visual acuity in $37.5 \%$ of patients. Conclusion: Bacteria, fungi and
\end{abstract}


acanthamoeba organisms were the microbiological isolates from the scrapings of corneal ulcer patients seen in the eye clinic of FETHA; with bacterial organisms being the most common. Farming activities, preceding eye trauma, delayed presentation, self-medication and use of traditional eye medications (TEM) were common findings among the patients. A future larger study is recommended to confirm the findings of this study. Eye health education campaigns should be directed at farmers to encourage early presentation to hospitals.

\section{Keywords}

Microbial Keratitis, Microbial Diagnosis, Corneal Ulcer, Predisposing Factors, Abakaliki

\section{Introduction}

Microbial keratitis is an important cause of corneal ulceration, a potentially blinding eye condition [1] [2]. The term microbial keratitis or suppurative keratitis refers to infection of the cornea by non-viral pathogens; and could be caused by bacteria, protozoa (e.g. acanthamoeba), fungi and microsporidia [2]. It is characterized by an acute or sub-acute onset of corneal inflammation with associated symptoms of redness, tearing, and pain in the affected eye with clinical findings of conjunctival injection, corneal ulceration associated with stromal inflammatory infiltrates. The healing of a corneal ulcer is usually associated with corneal scarring which causes corneal opacification and therefore visual loss (corneal blindness); depending on the size, location and depth of the corneal opacity [1] [2] [3] [4].

Corneal blindness is the second commonest cause of monocular blindness and the commonest cause of childhood blindness [1] [2] [3] [4]. Corneal blindness has also been described as a "silent epidemic" with the incidence in the developing world being 10 times more than in developed countries [4]. According to the Nigeria blindness and visual impairment survey of 2005-2007, corneal causes constitute $7.9 \%$ and $5.9 \%$ respectively of blindness and severe visual impairment [5]. In their report, Ashaye et al. [6] found that $26.7 \%$ of all corneal opacifications in Ibadan were caused by corneal ulcers while Dandona [7] reports 50\% in India. Apart from visual loss, corneal ulcers may lead to cosmetic blemish from the resultant scar as well as loss of the eyeball through evisceration [8].

The commonest predisposing factor for microbial keratitis in developed countries is contact lens use [9], while trauma is the commonest in the developing world [8] [9]. In Nigeria several predisposing factors have been reported, the commonest being trauma and traditional eye medication [10] [11] [12] [13]. The causative organisms of corneal ulcer differ significantly across regions and have been found to be influenced by climate and socio-economic factors [9] [13] [14] [15] [16]. In developed countries, viruses are the predominant aetiologic agents 
but among contact lens wearers, acanthamoeba is the commonest cause. Non-contact lens related acanthamoeba keratitis, although relatively rare, has been reported among agricultural workers and manual labourers, especially in developing countries with warmer weather and poor socioeconomic conditions, particularly following eye trauma and contamination with dirty water splash [17], Also, in the developing world, bacteria and fungi are reported as the predominant agents [12] [16] [17] [18] [19]. The outcome of treatment for microbial keratitis has been reported to be particularly poor in Africa [18]. Delayed presentation and delayed referral to health facilities where appropriate microbial diagnosis and treatment are available, have been highlighted as a major problem [18]. This study is aimed at determining the predisposing factors and predominant causative microbiological agents of corneal ulcers among the patients that present to the eye clinic of FETHA, with a view to formulating a protocol for managing corneal ulcers based on local evidence.

\section{Study Area}

This study was carried out at the department of ophthalmology of the Federal Teaching Hospital Abakaliki (FETHA). Abakaliki is the capital of Ebonyi state, one of the 36 states of Nigeria located in the South east region of Nigeria. It has a population of approximately 2.9 million people (projected from the 2006 national census) with a land mass covers 5530 square kilometers. The vegetation is a mixture of savanna and semitropical forest. Agriculture is the mainstay of the economy.

The Federal teaching hospital Abakaliki (FETHA) is the teaching hospital of the Ebonyi State University College of Health sciences. It is a product of the acquisition of the defunct Ebonyi State University Teaching Hospital (EBSUTH) Abakaliki by the Federal government in December 2011; and its merger with the defunct Federal Medical Centre (FMC) Abakaliki, to form a new institution which began full operations by February 2012. The department of Ophthalmology of FETHA is therefore relatively young in its present form and has continued to evolve in its role as a tertiary eye centre with the need to establish management protocols for various eye care needs of the catchment area, based on local evidence.

\section{Study Design}

This was a longitudinal descriptive study carried out over a 4-month period $\left(1^{\text {st }}\right.$ May to $30^{\text {th }}$ August 2015) at the Eye Clinic of the Department of Ophthalmology, Federal Teaching Hospital Abakaliki. All corneal ulcer patients who had clinical features of microbial keratitis and who consented to the study were recruited consecutively at diagnosis. Before recruitment, it was clearly explained to each patient that he/she was a research subject, and informed written consent obtained. Each patient was assured that they are free at any stage of the study to withdraw consent, and that withdrawal of consent would not in any way ad- 
versely affect his/her treatment. Confidentiality was maintained throughout the study. Ethical approval was obtained from the Health Research Ethics Committee of the Federal Teaching Hospital, Abakaliki.

\subsection{Corneal Sample Collection Procedures}

Corneal scraping was performed by an ophthalmologist using a sterile Bard-Parker blade size 15. The procedure was performed after instillation of $2 \%$ lignocaine hydrochloride. The specimens obtained from scraping the leading edge and base of each ulcer were swabbed with sterile swab stick and inoculated onto culture media including blood agar, chocolate agar and Sabourad Dextrose Agar (SDA). Scrapings were also directly smeared unto slides for staining and microscopy. On the first slide $10 \%$ potassium hydroxide $(\mathrm{KOH})$ was dropped on the smear and stained with lactophenol blue. Other smears were made and stained with Gram-stain, Giemsa-stain and modified Field-stain respectively. All the stained slides were examined microscopically for detection of microbial pathogens.

Specimens cultured on blood and chocolate agars for bacterial isolation were incubated aerobically at $37^{\circ} \mathrm{C}$ and evaluated at 24 hours, 48 hours and at 72 hours and then discarded if there were no growth; whereas the one inoculated onto SDA (supplemented with $2.5 \% \mathrm{v} / \mathrm{v}$ of chloramphenicol at concentration of $34 \mathrm{mg} / \mathrm{mL}$ ) for fungal isolation was incubated at room temperature and examined daily, and discarded after 7 days if no growth was present.

Microbial cultures were considered positive only if growth of the same organism was observed on at least two duplicate solid media plates. The specific identification of bacterial pathogens was based on colonial morphology, staining characteristics, and biochemical properties using standard microbiological procedure. Susceptibility testing of the bacterial isolate was also carried out in vitro with amikacin $(30 \mu \mathrm{g})$, chloramphenicol $(30 \mu \mathrm{g})$, ciprofloxacin $(5 \mu \mathrm{g})$, gatifloxacin $(5 \mu \mathrm{g})$, gentamycin $(10 \mu \mathrm{g})$, moxifloxacin $(5 \mu \mathrm{g})$, ofloxacin $(5 \mu \mathrm{g})$, and tobramycin $(10 \mu \mathrm{g})$ by the standard agar disc diffusion method (Kirby-Bauer).

Fungi were identified by their colony characteristics on SDA and by the morphological appearance in lactophenol cotton blue stain.

Acanthamoeba diagnosis was based on Giemsa and modified Field's staining techniques. The corneal ulcer scrapping transferred onto a clean grease free glass slide was allowed to air dry at room temperature. The dried slide was stained with $10 \%$ Giemsa stain and allowed for 20 minutes. Then stained smear was washed with distilled water and allowed to air dry at room temperature. The dried stained smear was examined microscopically using $\mathrm{x} 40$ and $\mathrm{x} 100$ objective lenses for 10 minutes spending about 1 minute per field. To confirm the detection of Acanthamoeba spp, another dried smear of corner ulcer was added immediately four drops of Field's stain B and 8 drops of Field's stain A. The slides were agitated until a golden scum appeared on the surface of the smear within 15 $\mathrm{s}$. The slides were rinsed for $2 \mathrm{~s}$ under a stream of water and allowed to dry at room temperature (37). The smear was examined microscopically using $\times 40$ 
and $\times 100$ objective lenses for 10 minutes spending about 1 minute. Other laboratory tests Blood samples were also collected from all the patients for fasting blood sugar and HIV screening respectively.

\subsection{Data Management}

Data collection was done using an interviewer administered questionnaire filled by the evaluating Ophthalmologist. Information obtained included socio-demographic data, presenting symptoms, duration of symptoms before presentation, history of trauma and use of TEM. Visual acuity at presentation and discharge were also noted. Data collected was transferred from the questionnaire into a personal laptop computer and analyzed using the Statistical Package for Social Sciences (SPSS), version 23 (SPSS Inc, Chicago, Illinois, USA), and reported as frequency distributions, percentages and means \pm standard deviation.

\subsection{Clinical Management}

Following sample collection, each patient was commenced on broad-spectrum antibiotics (ceftazidine as subconjunctival injection-100 $\mathrm{mg}$ daily for 3 days and as eye drops $50 \mathrm{mg}$ per $\mathrm{ml}$ ), , ciprofloxacin as eye drops), antifungal agents (natamycin) amidst other supportive treatment such as $1 \%$ atropine eye drops and analgesics, pending the laboratory microbial diagnosis and result of culture and sensitivity to guide any change in medications. Due to unavailability of specific acantamoeba medications such as chlorhexidine ophthalmic drops, polyhexamethylene biguanide (PHMB), propamidine (brolene) or hexamidine (desmodine); we added providone iodine $2.5 \%$ to the protocol for treatment of patients with a microbial diagnosis of acantamoeba.

\section{Results}

Within the study period of 4 months, 8 out of the 852 patients that visited the Eye Clinic of the Federal Teaching Hospital Abakaliki were diagnosed with corneal ulcers (a hospital prevalence rate of 0.59\%). There were 5 males and 3 females. The ages ranged from 24 to 74 years with a mean age of $47.1 \pm 20.3$. Majority $(62.5 \%)$ of the patients were farmers.

\subsection{Predisposing Factors}

All patients presented late after unsuccessful attempts at treatment. The mean duration before presentation was 2 weeks. A history of recent corneal injury or trauma with wood/vegetative material was obtained in 4 (50\%) patients. The use of traditional eye medications (TEM) was common. All cases of spontaneous red eye were mismanaged either by the use of TEM or intervention by quacks/self. Relevant systemic findings were HIV positive status in 1 (12.5\%) patient and diabetes mellitus in one patient (12.5). See Table 1.

\subsection{Microbiological Diagnosis}


Corneal scrapings in this series yielded microbial diagnosis in 7 (87.5\%) of the Table 1. Socio-demographic and clinical characteristics of the patients.

\begin{tabular}{|c|c|c|}
\hline Variables & Frequency $(\mathrm{n}=8)$ & Percentage \\
\hline \multicolumn{3}{|l|}{ Age: } \\
\hline Mean age & $47.1 \pm 20.3$ & \\
\hline Age Range: & 50 & \\
\hline Minimum Age & 24 & \\
\hline Maximum Age & 74 & \\
\hline \multicolumn{3}{|l|}{ Gender: } \\
\hline Male & 5 & 62.5 \\
\hline Female & 3 & 37.5 \\
\hline \multicolumn{3}{|l|}{ Occupation: } \\
\hline Farmer & 5 & 62.5 \\
\hline Student & 2 & 25 \\
\hline Commercial Motorcyclist & 1 & 12.5 \\
\hline \multicolumn{3}{|l|}{ Predisposing Activity: } \\
\hline Trauma & 4 & 50 \\
\hline Red Eye (spontaneous) & 4 & 50 \\
\hline \multicolumn{3}{|l|}{ Duration before presentation in days: } \\
\hline Minimum & 7 & \\
\hline Maximum & 21 & \\
\hline Mean duration & $14.5 \pm 6.0$ & \\
\hline Self Medication before Presentation & 8 & $100 \%$ \\
\hline \multicolumn{3}{|l|}{ Use of Traditional Eye Medication } \\
\hline yes & 5 & 62.5 \\
\hline No & 3 & 37.5 \\
\hline${ }^{*} \mathrm{HIV}$ positive & 1 & 12.5 \\
\hline Diabetes Mellitus & 1 & 12.5 \\
\hline
\end{tabular}

*Facility for HIV viral load estimation was not available.

patients, out of which 4 (50\%) were positive for bacteria and $2(25 \%)$ had a fungal isolate. The bacterial isolates were identified to be Staphylococcus aureus while the fungal isolates were Fusarium spp. and aspergillus species respectively. Acanthamoeba was the microscopy diagnosis in 2 patients (25\%), with no culture growth. None of the patients ever used contact lenses. See Table 2.

\subsection{Treatment Outcome in Affected Eyes}

All eight patients had their diagnosis in one eye only. All affected eyes were blind at presentation at $(\mathrm{VA}<3 / 60)$. After treatment, the visual acuity improved in 3 (37.5\%) of the patients among which and 2 of these eyes were no longer considered blind. See Table 3. There was $100 \%$ success with preservation of all the eyes as there was no corneal perforation, or need for evisceration.

\section{Discussion}

The slight male preponderance $(62.5 \%)$ in this study perhaps reflects the greater involvement of men in outdoor activities (including farming) in this region than 
women. This finding is in keeping with the study by Oladigbolu [16] in Northern Table 2. Microbiological diagnosis.

\begin{tabular}{lcc}
\hline Isolated Microbes & Frequency & Percent \\
\hline Staphylococcus aureus & 3 & 37.5 \\
Acanthamoeba species & 2 & 25.0 \\
Aspergillus species & 1 & 12.5 \\
Fusarium Species & 1 & 12.5 \\
No growth & 1 & 12.5 \\
Total & 8 & 100.0 \\
\hline
\end{tabular}

Table 3. Visual acuity and treatment outcome in affected eyes.

\begin{tabular}{ccc}
\hline Variables & Frequency $(\mathrm{n}=8)$ & Percentage \\
\hline VA on Presentation: & 2 & 25 \\
PL & 5 & 62.5 \\
HM & 1 & 12.5 \\
$1 / 60$ & & \\
& & 37.5 \\
Final VA on discharge & 3 & 37.5 \\
PL & 3 & 12.5 \\
HM & 1 & 12.5 \\
$3 / 60$ & 1 & \\
$6 / 60$ & & 37.5 \\
Visual Improvement after treatment & & 62.5 \\
Yes & 3 & \\
No & 5 & \\
Complications: & & \\
Corneal Perforations & Nil & \\
Evisceration & Nil & \\
\hline
\end{tabular}

VA: Visual acuity; HM: Hand Motion; PL: Perception of Light.

Nigeria and other reports from Ghana and India [19] [20]; but differs from Ezegwui's finding of slight female preponderance in South East Nigeria [15]. It is believed that increased physical/outdoor activities as either occupation or sport may increase the risk of corneal ulceration as reported by Basak [20]. However the trends are changing, with women getting more involved in farm-related and other outdoor activities

Occupation was also shown to be an important predisposing factor in this study as majority of the patients were farmers (62.5\%). This is not a surprise, knowing that agricultural workers are usually exposed to trauma during agricultural activities especially those involving cutting of wood while clearing the forest or cutting vegetable supplies for animals. Other studies have shown that labourers/farmers contribute $54 \%-70 \%$ of corneal ulcer patients [17]-[25]. Ebonyin State has been known to have a large population of peasant farmers especially in the older generations. In this study, the patients aged $\geq 60$ years of age and were all farmers. The younger generations are tending towards pursuing education and more modern career paths. Farmers have been documented to be 
at risk of corneal injury with vegetative matter (trauma) [18]-[24].

The finding of preceding ocular trauma in $50 \%$ of patients in this study is similar to Oladigbolu's report of $51.3 \%$ in Nigeria [17] and that by Talukder et al. [24] of 56.9\% in Bangladesh but less than the $82.9 \%$ in West Bengal [20]. Corneal injury is a known predisposing factor for microbial keratitis [8] [9] [10] [11] [14] [15] [17] [20]-[25].

The use of traditional eye medications (TEM) is still a common practice, as most patients in Africa consult a traditional healer before presentation to a hospital [25]. In this study, the use of TEM prior to presentation was noted in $50 \%$ of patients, demonstrating the high patronage of traditional healers in our locality, the rest of the patients had tried other forms of alternative medications, self medication and quackery. This agrees with reports by Chhangte [22] showing that $88.1 \%$ of patients had consulted such alternative places prior to hospital presentation. In our study, all our patients (100\%) had tried self-medication, chemists, TEM or quacks before presentation. This practice is corroborated by other studies from Nigeria and other developing countries [10] [11] [14] [15] [17] [20]-[26]. In a previous study done in the present locality (Ebonyi state) of Nigeria on choice of care giver for ocular symptoms in general, Ogbonnaya et al. [26] noted that the majority $68 \%$ of respondents preferred to patronize the patent medicine vendors ("Chemist") and/or traditional healers who offer them eye drops "capsule powder" and other forms of alternative medicine rather than visiting available health centres for eye care. The local preparations obtained from these cadres of care givers may themselves be the source of the microorganisms causing the microbial keratitis as well as toxic injuries (chemical burn) that predisposes the patient to the corneal ulceration.

The time of presentation of patients to hospital following symptoms reflects the health-seeking behavior of patients in developing countries. Late presentation is reported by most studies from Nigeria and other developing countries [10] [15] [17] [20]-[26]. In South Eastern India where most eye medications are sold over the counter without a prescription, delayed presentation, probably after self medication, has been documented to adversely affect treatment outcome for corneal ulcers [22] [27]. Delay in presentation in this series ranged from 1 to 3 weeks with an average of 2 weeks between onset of symptoms and presentation to hospital. Delayed presentation has been reported severally [12]-[18]. Self-medication, patronizing quacks, use of traditional medication all contribute to the delayed presentation with patients reporting to the hospital only after failure of other modalities have been established Oladigbolu et al. [16]. All the patients presented blind in the affected eye with visual acuity ranging from light perception to counting fingers at 1 metre.

The high culture sensitivity of the corneal scrapings in this study (87.5\%) of the corneal scrapings is in keeping with other reports from Ghana [19] South India [17] and eastern India [20]; which reported culture positivity of $57.3 \%$, $70.6 \%$ and $67.7 \%$ respectively. Bacterial keratitis in ths study was most common 
(37.5\%) followed by fungal keratitis. Most other studies from Africa and other tropical countries report a predominance of fungal keratitis [18] [19] [20] [21]. The finding of acanthamoeba in $25 \%$ of cases was unexpected as none of the patients used contact lenses. However there have been some reports of culture-positive acanthamoeba keratits among non-contact lens wearers [17] [27]. Perhaps the common practice in this region of self-medication with substances and TEM before presentation to hospital may be the source of this organism as it is ubiquitous in most water sources and could constitute a common contaminant of traditional eye medications and quack preparations.

Age and occupation have been noted to be significant predictors of the likely organism or microbial growth to expect from a corneal scrapping. In their study Kibret and Bitew [21] noted that the age group 20 - 50 years was the commonest for bacterial ulcers while the older age group 50 - 70 years and farming as occupation, had more of fungal keratitis [21]. This finding agrees with the present study where fungal isolates were found in the older patients, who were both farmers.

Poor visual outcome from microbial keratitis is a big problem in Africa [18]. Unavailability of appropriate antibiotics and particularly ophthalmic antifungal and anti acanthamoeba agents is a serious problem. Furthermore the practice of first consulting the traditional healer not only results in further delay but also compounds the ulcers by further contamination or toxic injury.

\section{Conclusion and Recommendation}

The microbial organisms isolated from the corneal ulcer patients seen at Federal Teaching Hospital Abakaliki were bacterial, fungal and acanthamoeba, with bacterial keratitis being most common. Farming activities, preceding eye trauma, delayed presentation, self-medication and use of traditional eye medications (TEM) were common findings among the patients. Revision of empirical treatment protocols based on findings of this study is recommended. A future larger study is also recommended using lessons from this study. Eye health education should be directed at farmers to encourage early presentation to hospitals and the use of protective spectacles.

\section{Conflicts of Interest}

The authors declare no conflicts of interest regarding the publication of this paper.

\section{Limitations of the Study}

Limitations of this study are the short duration and the small number of patients.

\section{References}

[1] Srinivasan, M., Gonzales, C.A., George, C., Cevallos, V., Mascarenhas, J.M., Asokan, 
B., et al. (1997) Epidemiology and Aetiological Diagnosis of Corneal Ulceration in Madurai, South India. British Journal of Ophthalmology, 81, 965-971. https://doi.org/10.1136/bjo.81.11.965

[2] Tuft, S. and Burton, M. (2013) Microbial Keratitis. Royal College of Ophthakmologists, London.

[3] Whitcher, J.P., Srinivasan, M. and Upadhyay, M.P. (2001) Corneal Blindness: A Global Perspective. Bulletin of the World Health Organization, 79, 214-221.

[4] Whitcher, J.P. and Srinivasan, M. (1997) Corneal Ulceration in the Developing World-A Silent Epidemic. British Journal of Ophthalmology, 81, 622-623.

[5] Abdull, M.M., Sivasubramaniam, S., Murthy, G.V., Gilbert, C., Abubakar, T., Ezelum, C., Rabiu, M.M. and the Visual Impairment Study Group (2009) Causes of Blindness and Visual Impairment in Nigeria: The Nigeria National Blindness and Visual Impairment Survey. Investigative Ophthalmology \& Visual Science, 50, 4114-4120. https://doi.org/10.1167/iovs.09-3507

[6] Ashaye, A.O. and Oluleye, T.S. (2004) Pattern of Corneal Opacity in Ibadan, Nigeria. Annals of African Medicine, 3, 185-187.

[7] Dandona, L., Ragu, K., Janarthanan, M., Naduvilath, T.J., Shenoy, R. and Rao, G.N. (1997) Indications for Penetrating Keratoplasty in India. Indian Journal of Ophthalmology, 45, 163-168.

[8] Hongyok, T. and Leelaprute, W. (2016) Corneal Ulcer Leading to Evisceration or Enucleation in a Tertiary Eye Care Center in Thailand: Clinical and Microbiological Characteristics. Journal of the Medical Association of Thailand, 99, S116-S1122.

[9] Ibrahim, Y.W., Boase, D.L. and Cree, I.A. (2009) Epidemiological Characteristics, Predisposing Factors and Microbiological Profiles of Infectious Corneal Ulcers: The Portsmouth Corneal Ulcer Study. British Journal of Ophthalmology, 93, 1319-1324. https://doi.org/10.1136/bjo.2008.151167

[10] Dawodu, O.A., Osahon, A.I. and Emifoniye, E. (2003) Prevalence and Causes of Blindness in Otibhor Okhae Teaching Hospital, Irrua, Edo State, Nigeria. Ophthalmic Epidemiology, 10, 323-330. https://doi.org/10.1076/opep.10.5.323.17325

[11] Al-Otabi, A.G. (2012) Non-Viral Microbial Keratitis in Children. Saudi Journal of Ophthalmology, 26, 191-197. https://doi.org/10.1016/j.sjopt.2011.10.002

[12] Ezegwui, I.R. (2010) Corneal Ulcers in a Tertiary Hospital in Africa. Journal of the National Medical Association, 102, 644-646. https://doi.org/10.1016/S0027-9684(15)30642-8

[13] Green, M., Apel, A. and Stapleton, F. (2008) Risk Factors and Causative Organisms in Microbial Keratitis. Cornea, 27, 22-27. https://doi.org/10.1097/ICO.0b013e318156caf2

[14] Panda, A., Satpathy, G., Nayak, N., Kumar, S. and Kumar, A. (2007) Demographic Pattern, Predisposing Factors and Management of Ulcerative Keratitis: Evaluation of One Thousand Unilateral Cases at a Tertiary Care Centre. Clinical \& Experimental Ophthalmology, 35, 44-50. https://doi.org/10.1111/j.1442-9071.2007.01417.x

[15] Olawoye, O.O., Bekibele, C.O. and Ashaye, A.O. (2011) Suppurative Keratitis in a Nigerian Tertiary Hospital. Nigerian Journal of Ophthalmology, 19, 27-29. https://doi.org/10.4314/njo.v19i1.70774

[16] Oladigbolu, K., Rafindadi, A., Abah, E. and Samaila, E. (2013) Corneal Ulcers in a Tertiary Hospital in Northern Nigeria. Annals of African Medicine, 12, 165-170. https://doi.org/10.4103/1596-3519.117626

[17] Bharathi, M.J., Ramakrishnan, R., Vasu, S., Meenakshi, R., Shivkumar, C. and Pala- 
niappan, R. (2003) Epidemiology of Bacterial Keratitis in a Referral Centre in South India. Indian Journal of Medical Microbiology, 21, 239-245.

[18] Burton, M.J., Pithuwa, J., Okello, E., Afwamba, I. and Onyango, J.J., Oates, F., Chevallier, C. and Hall, A.B. (2011) Microbial Keratitis in East Africa: Why Are the Outcomes So Poor? Ophthalmic Epidemiology, 18, 158-163. https://doi.org/10.3109/09286586.2011.595041

[19] Leck, A.K., Thomas, P.A., Hagan, M., Kaliamurthy, J., Ackuaku, E., John, M., Newman, M.J., Codjoe, F.S., Opintan, J.A., Kalavathy, C.M., Essuman, V., Jesudasan, C.A. and Johnson, G.J. (2002) Aetiology of Suppurative Corneal Ulcers in Ghana and South India, and Epidemiology of Fungal Keratitis. British Journal of Ophthalmology, 86, 1211-1215. https://doi.org/10.1136/bjo.86.11.1211

[20] Basak, S.K., Basak, S., Mohanta, A. and Bhowmick, A. (2005) Epidemiological and Microbiological Diagnosis of Suppurative Keratitis in Gangetic West Bengal, Eastern India. Indian Journal of Ophthalmology, 53, 17-22.

https://doi.org/10.4103/0301-4738.15280

[21] Kibret, T. and Bitew, A. (2016) Fungal Keratitis in Patients with Corneal Ulcer Attending Minilik II Memorial Hospital, Addis Ababa, Ethiopia. BMC Ophthalmolo$g y, 16,148$. https://doi.org/10.1186/s12886-016-0330-1

[22] Chhangte, L., et al. (2015) Epidemiological and Microbiological Profile of Infectious Corneal Ulcers in Tertiary Care Centre, Kumaon Region, Uttarakhand Dr.

[23] Ibrahim, M.M., Vanini, R., Ibrahim, F.M., Martins Wde, P., Carvalho, R.T., Castro, R.S., et al. (2011) Epidemiology and Medical Prediction of Microbial Keratitis in Southeast Brazil. Arquivos Brasileiros de Oftalmologia, 74, 7-12. https://doi.org/10.1590/S0004-27492011000100002

[24] Talukder, A.K., Sultana, Z., Jahan, I., Khanam, M., Bhuiyan, S.I. and Rahman, M.B. (2016) Management of Infective Corneal Ulcer: Epidemiology Needs to Be Evaluated as Priority Basis. Mymensingh Medical Journal, 25, 415-420.

[25] Ukponmwan, C.U. and Momoh, N. (2010) Incidence and Complications of Traditional Eye Medications in Nigeria in a Teaching Hospital. Middle East African Journal of Ophthalmology, 17, 315-319. https://doi.org/10.4103/0974-9233.71596

[26] Ogbonnaya, C.E. and Ogbonnaya, L.U. (2016) Choice of Eye Care Giver in a Rural Community in Ebonyi State. Journal of Medical Science and Clinical Research, 4, 12908-12914. https://doi.org/10.18535/jmscr/v4i10.06

[27] Sharma, S., Garg, P. and Rao, G. (2000) Patient Characteristics, Diagnosis, and Treatment of Non-Contact Lens Related Acanthamoeba keratitis. British Journal of Ophthalmology, 84, 1103-1108. https://doi.org/10.1136/bjo.84.10.1103 